\title{
Cardiac Interoception in Infants: Behavioral and Neurophysiological Measures in Various Emotional and Self-Related Contexts
}

\author{
Marieke L. Weijs ${ }^{1}$, Moritz M. Daum ${ }^{1,2}$, Bigna Lenggenhager ${ }^{1}$ \\ ${ }^{1}$ Department of Psychology, University of Zurich, Zurich, Switzerland \\ ${ }^{2}$ Jacobs Center for Productive Youth Development, University of Zurich, Switzerland
}

\begin{abstract}
Author Note
Corresponding author: Marieke Weijs, Department of Psychology, University of Zurich Binzmühlestrasse 14, Box 9, marieke.weijs@uzh.ch Conflict of interest statement: The authors report no conflict of interest. Acknowledgements: This research was supported by the Swiss National Science Foundation (grant number: PP00P1_170511). We thank Fiona Pugin and Peyman Khodadust for assistance with the experimental setup, Leonie Hartmann for help with coding the videos, and Raphaela Schnider for help with recruitment. Thanks to the participants and parents for taking part in this study.
\end{abstract}




\begin{abstract}
Interoception, the perception of internal bodily signals, is fundamental to our sense of self. Even though theoretical accounts suggest an important role for interoception in the development of the self, empirical investigations are limited, in particular, in infancy. Previous studies used preferential-looking paradigms to assess the detection of sensorimotor and multisensory contingencies in infancy, usually related to proprioception and touch. So far, only one single recent study reported that infants discriminated audiovisual stimuli that were presented synchronous or asynchronous with their heartbeat. This discrimination was related to the amplitude of the infant's heartbeat evoked potentials (HEP), a neural correlate of interoception. In the current study, we measured looking preferences between synchronous and asynchronous visuocardiac (bimodal), and audiovisuocardiac (trimodal) stimuli as well as the HEP in conditions of different emotional contexts and with different degrees of selfrelatedness in a mirror-like setup. While the infants preferred trimodal to bimodal stimuli, we did not observe the predicted differences between synchronous and asynchronous stimulation. Furthermore, the HEP was not modulated by either the emotional context or by the self-relatedness. These findings contradict previously published findings and highlight the need for further studies on the early development of interoception in relation to the development of the self.
\end{abstract}

Keywords: heartbeat evoked potential, contingency, self-perception, bodily self development 


\section{Cardiac Interoception in Infants: Behavioral and Neurophysiological Measures in Various Emotional and Self-Related Contexts}

Interoception, the sense of the physiological state of the body (Craig, 2002), is both fundamental to maintenance of homeostasis (Petzschner, Garfinkel, Paulus, Koch, \& Khalsa, 2021), and linked to cognition, emotion, and the sense of self (Azzalini, Rebollo, \& TallonBaudry, 2019). Theoretical accounts attribute an important role to interoception in early development of self-awareness and social cognition, stating that awareness of one's own internal bodily signals might give rise to self-regulatory behaviors, and social interactions necessary for the maintenance of homeostasis in early life (Ciaunica \& Crucianelli, 2019; Filippetti, 2021; Fotopoulou \& Tsakiris, 2017; Montirosso \& McGlone, 2020; Mundy \& Jarrold, 2010). It is thought that early development of self-awareness, which requires a distinction between the self and the other or the environment, relies on the perception and integration of contingent multisensory information (Bahrick, 2013; Jacquey, Fagard, Esseily, \& O'Regan, 2020). It has been shown that infants are sensitive to various multimodal contingencies between the environment and their own body, such as visuo-tactile, and visuoproprioceptive synchrony from early on (Bahrick \& Watson, 1985; Filippetti, Johnson, Lloyd-Fox, Dragovic, \& Farroni, 2013; Zmyj, Jank, Schütz-Bosbach, \& Daum, 2011). Along these lines, it has been suggested that infants also detect contingencies between cardiac and audiovisual signals (Maister, Tang, \& Tsakiris, 2017).

In adults, a close link of interoception and self-awareness has been proposed (Tsakiris, 2017) and empirical studies showed that focusing on oneself, for example by looking in the mirror or at a picture of oneself, improves performance in a heartbeat tracking tasks (Ainley, Maister, Brokfeld, Farmer, \& Tsakiris, 2013; Ainley, Tajadura-Jiménez, Fotopoulou, \& Tsakiris, 2012). Similar to the behavioral measures of interoception, the neurophysiological heartbeat evoked potential (HEP) has found to be modulated in response 
to self-related (Babo-Rebelo, Buot, \& Tallon-Baudry, 2019; Petzschner et al., 2019), as well as emotional stimuli (Gentsch, Sel, Marshall, \& Schütz-Bosbach, 2019; Luft \& Bhattacharya, 2015). The HEP is a cortical potential that occurs $200-650 \mathrm{~ms}$ after the R-peak in frontocentral regions, and is thought to reflect interoceptive processing at a cortical level (Coll, Hobson, Bird, \& Murphy, 2021; Park \& Blanke, 2019). Alterations in HEP have also been found in several clinical conditions related to both alterations of the self (e.g. Schulz et al., 2015), and of emotional processing (e.g. Flasbeck, Popkirov, Ebert, \& Brüne, 2020). Developmental investigations of the HEP are still limited, but evidence for a relation of the HEP with both a behavioral measure of interoceptive sensitivity, and emotional processing in infants has been reported (Maister et al., 2017). More specifically, the HEP amplitude was larger when infants observed negative as compared to neutral or positive emotions.

Additionally, the HEP amplitude positively correlated with interoceptive sensitivity, defined as the difference in looking time between synchronous and asynchronous audiovisuocardiac stimuli.

In the current study, we investigated the relation between cardiac interoception, emotion, and early self-awareness. With this, we aimed to replicate and extend the previous findings by Maister and colleagues (2017). Similar to this previous study we assessed interoceptive sensitivity using looking-time measures to external stimuli presented in or out of synchrony with the infant's heartbeat in the first part of the experiment, and expected previously found longer looking times for asynchronous stimulation. To further assess whether cardiac discrimination ability depends on sensory modality of the contingency and the richness of the sensory information, we compared looking times between synchronous and asynchronous stimuli in a trimodal (audiovisuocardiac) condition, as in the original study, and added a bimodal (visuocardiac) condition, to test whether redundant sensory information is indispensable to contingency detection in a cardiac interoception task. 
Bimodal visuocardiac stimuli have been used previously to alter self-awareness in adults as a manipulation of contingencies between interoceptive and exteroceptive signals (Aspell et al., 2013; Heydrich et al., 2018), we thus expected visuocardiac stimulation only to induce the same effects as trimodal audiovisuocardiac stimulation. In line with previous studies we formulate the following expectations: First, we expected the infants to look longer in the trimodal condition than the bimodal condition. Infants have been shown to be more interested and learn better from intersensory redundancy (Bahrick \& Lickliter, 2000, 2012). Second, we expected infants to look longer towards asynchronous than synchronous stimuli (Maister et al., 2017). We had no specific expectations about a potential interaction of synchrony and modality, but such interaction would suggest that redundancy additionally affects synchrony detection.

In the second part of the experiment that was conducted at a different day with the same participants, we concurrently measured ECG and EEG to assess the HEP in contexts varying either in an emotional context or self-relatedness. The aim was twofold again: First, we wanted to replicate the previously found alteration in the HEP in infants depending on the emotional context (Maister et al., 2017), and second, we aimed to test whether a modulation of the HEP could be found by alteration of self-related contexts. Emotional context has previously been shown to modulate interoceptive processes (Critchley \& Garfinkel, 2017), for example through modulation of the HEP both in adults (e.g. Gentsch et al., 2019), as well as in infants (Maister et al., 2017). The latter showed that the HEP amplitude was be larger when infants observed videos of faces expressing negative, as compared to positive or neutral emotions. We thus presented angry compared to happy faces and expected larger HEP amplitudes when infants looked at angry faces. In line with the previous study, we also expected a positive correlation of cardiac discrimination, quantified as the difference in 
looking time between synchronous and asynchronous stimuli, with the HEP amplitude averaged across all experimental conditions.

To extend the theoretical discussion on how interoception relates to perception of exteroceptive multisensory contingencies, we extended the EEG paradigm with a self-related context. Here, we displayed synchronous and asynchronous webcam recordings of the infant, as infants have shown to be sensitive to visuoproprioceptive contingencies from at least 4months old (Rochat \& Striano, 2002). It is thought that this awareness of multisensory contingencies is an implicit precursor to explicit mirror self-recognition between 18 and 24 months (Amsterdam, 1972; Rochat \& Botto, 2021). This is further corroborated by behavioral findings showing that toddlers first only pass the mirror self-recognition task when mirror displays are synchronous, and only later pass it when the display is asynchronous (Miyazaki \& Hiraki, 2006). In adults, self-observation in a mirror has been shown to increase interoceptive sensitivity (Ainley et al., 2012), and the HEP was differently modulated when thinking about the self as compared to others (Babo-Rebelo et al., 2019). We thus assumed that self-observation with visuomotor synchrony, would enhance basic selfawareness and self-other distinction in infants, even before the onset of explicit mirror selfrecognition. In turn, we expected this to be reflected in an increased HEP amplitude, as compared to asynchronous videos. Finally, we assessed how interoception, as measured behaviorally and neurophysiologically, relates to infant's temperament, as measured with the IBQ-R (Putnam, Helbig, Gartstein, Rothbart, \& Leerkes, 2014), to explore whether there is a relation between behavior and interoception already at five months of age.

\section{Methods}

\section{Participants}

Behavioral data of 42 infants aged between 5 to 7 months were recorded, of which 31 infants (17 female, age $M=186$ days, $S D=15$ days) were included in the final dataset for the 
behavioral task. The other 11 infants were excluded because they became fussy before or during the task and did not complete enough trials (see inclusion criteria below). For the EEG session, 3 appointments had to be cancelled because of a measles outbreak at the department, and 1 because of illness, meaning that 38 participants returned for the second session. Out of these, three datasets were excluded because of technical issues during the recording, 5 because of fussiness during or after application of the EEG cap, which yielded a final EEG sample of 29 infants ( 15 female, age $M=191$ days, $S D=16$ days). In total, 22 infants (11 female, age $M=188$ days, $S D=17$ days) provided useable data for both the behavioral and EEG session needed for the correlational analyses. Infants were recruited via a database of parents who volunteered to participate in developmental studies. All were born full term (gestational age $\geq 37$ weeks) and had normal birth weight ( $\geq 2500 \mathrm{~g}$ ). Infants received a certificate and gift worth approximately CHF 5,- as compensation for participation. Parents provided informed consent prior to participation of the study. The study was approved by the Cantonal Ethics Committee of Zurich (NR: 2018-00485), and was conducted in accordance with the ethical standards of the Declaration of Helsinki.

\section{Procedure}

The experiment was initially planned as a longitudinal study testing children at the age of 6, 18 and 24 months. However, due to COVID the study had to be interrupted and only the first timepoint was recorded. The experiment thus consisted of two sessions, which were maximally 7 days apart. The first session consisted of the looking-time task, the second session of the EEG recording.

\section{Looking-Time Task}

This task followed a $2 \times 2 \times 2$ mixed design. Modality, either bimodal (visuocardiac) or trimodal (audiovisuocardiac), and Synchrony, either synchronous or asynchronous, were manipulated within participants. Finally, Speed of the asynchronous stimuli, faster $(110 \%$ of 
the recorded heartbeat) or slower $(90 \%)$ was manipulated between participants to avoid exclusively measuring a preference for speed rather than for contingency. Modality was presented in a blocked design, with either bimodal or trimodal first in counterbalanced order. Each block consisted of maximally six trials, where each trial was a consecutive presentation of a synchronous display and an asynchronous display (see Figure 1). Each trial was followed by the grasping preference test. The blocks were stopped early in case the infant became fussy or completely lost attention. Infant's behavior and the screen were video-recorded with three cameras for later offline analyses. One camera was placed directly above the screen to record the direction of gaze, a second one filmed the infant from the top, to assess grasping responses. A third camera was directed towards the screen, to be able to synchronize the looking-time coding with the stimulus presentation.

\section{Figure 1}

Procedure of the Behavioral Task
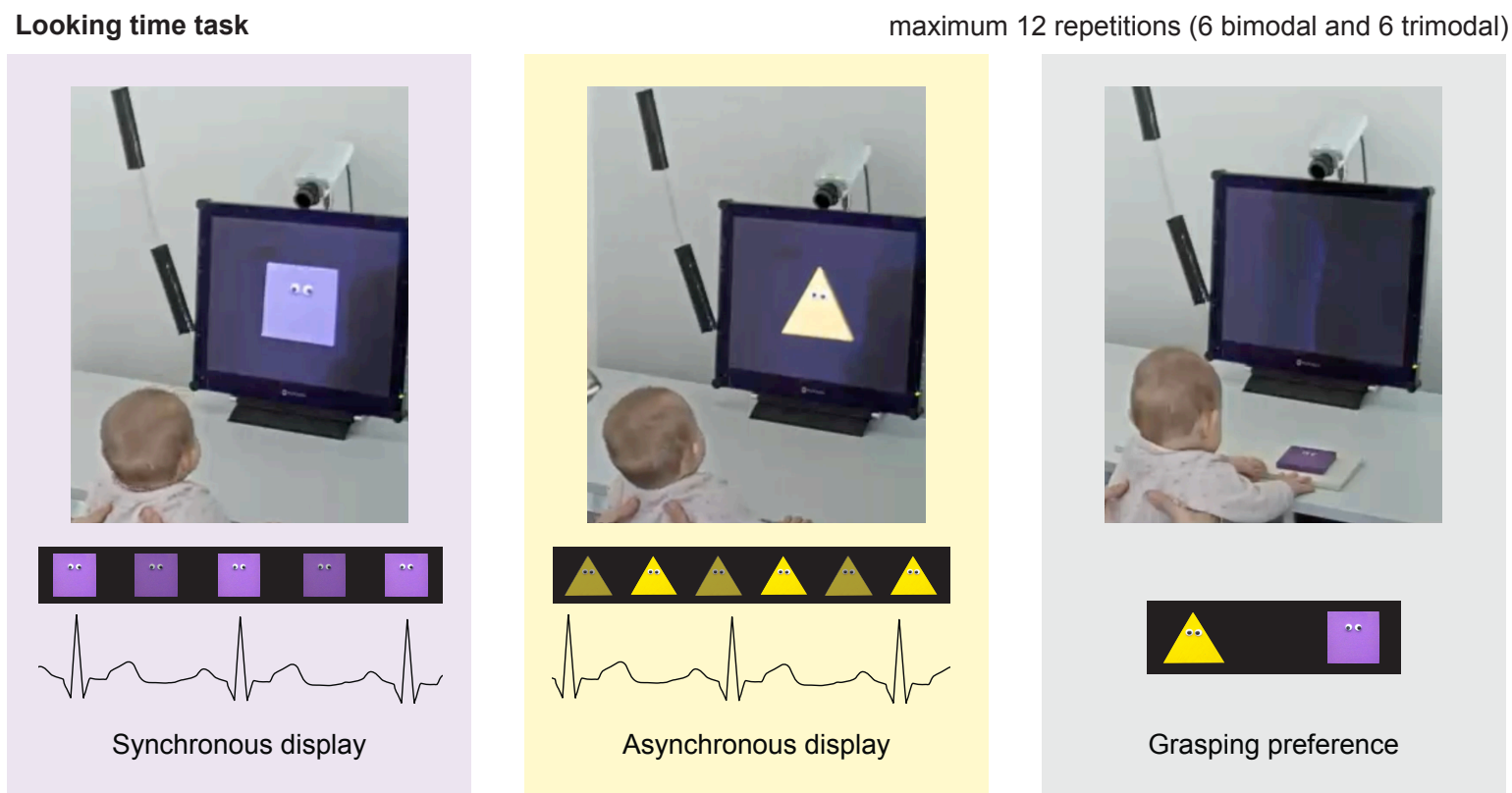

Note. Infants were seated in front of the screen and were presented with stimuli that blinked in synchrony with the heartbeat (purple) or in asynchrony (yellow). After a synchronous and 
asynchronous stimulus presentation, there was a grasping trial where the two figures were presented on a wooden board in front of the infant, and the infant reached for either stimulus.

Stimulus Presentation. During the looking-time task, coloured geometric shapes were presented on a computer screen placed approximately $50 \mathrm{~cm}$ in front of the infant, who was seated on the parent's lap. Stimulus presentation was synchronized with the heartbeat. To this end, ECG was recorded with a Biopac MP150 and ECG100C amplifier (Biopac Systems Inc, Goleta, CA, USA) from $3 \mathrm{Ag} / \mathrm{AgCl}$ electrodes (Servoprax GmBh, Wesel, Germany) placed on the infant's chest and abdomen. Data were stored using Acqknowledge software at a sampling rate of $500 \mathrm{~Hz}$ (Biopac Systems Inc, Goleta, CA, USA). The built in R-wave detection mode of the ECG100C amplifier was used, which detects R-peaks online and outputs smoothed R-wave peaks. This mode is more robust against movement artefacts than a complete ECG recording would be. Each time an R-wave was detected, a parallel-port trigger was sent from the Biopac to the stimulus presentation computer. This trigger was detected in MATLAB and elicited a stimulus presentation. Each trial started with an auditory attention grabber, saying "lueg emol", the Swiss German word for "look". After $1 \mathrm{~s}$, the stimulus presentation started for a duration of $20 \mathrm{~s}$. In the trimodal condition, the presentation of the blinks was accompanied by an acoustic sound signal, a beep. These signals were triggered by the ECG R-peak in the synchronous condition. In the asynchronous condition, the speed of the asynchronous blinking was determined by a prerecording of three interbeat intervals, preceding the onset of each trial. Then, the average of three interbeat intervals was calculated and multiplied by 0.9 (for faster) or 1.1 (for slower) to determine the speed of the blinking in the asynchronous trial. After the synchronous and asynchronous stimulus both finished, the same figures as were shown on the screen were presented in a wooden $3 \mathrm{D}$ model front of the infant on a wooden board to assess grasping preference. Grasping preference are not reported 
here due to a flaw in the setup that we only became of during the data analysis process. The stimuli were always presented from the same side, biasing grasps strongly towards the first stimulus that came into the infant's field of view.

Measures. In the looking-time task, the preference for synchronous or asynchronous stimuli was assessed by the infants' looking preference. Looking time was coded as the time that the infant's gaze was directed at the screen while the stimuli were presented on screen.

\section{EEG Task}

EEG recordings were performed in a dimly lit, sound attenuated, and electrically shielded room. The infants were seated on the lap of the caregiver at a distance of approximately $60 \mathrm{~cm}$ from a 17 -inch monitor. A black cloth covered the caregivers' body, as to mask out any movements from the caregiver. The infant was filmed with a webcam, which was positioned so that the infants full face and body were in view, but the caregiver's face was out of view. In counterbalanced order, three blocks of each emotional condition (happy and angry), and three blocks of each self-related condition (self-synchronous and selfasynchronous) were presented. Emotional faces were taken from the Montréal Pain and Affective Face Clips (Simon, Craig, Gosselin, Belin, \& Rainville, 2008). Clips from four female and four male actors were selected, each face appeared on the screen for $2 \mathrm{~s}$, where the emotion was displayed at $50 \%$ intensity, then gradually changing to $100 \%$, and back to $50 \%$. Videos for the happy and angry condition were edited to a length of $20 \mathrm{~s}$. For the selfsynchronous condition, the recording from the webcam was displayed on the screen in real time for $20 \mathrm{~s}$. For the self-asynchronous condition, the recording was delayed with $3 \mathrm{~s}$ and then played back for $20 \mathrm{~s}$. 


\section{Figure 2}

Procedure and Stimuli of the EEG Task
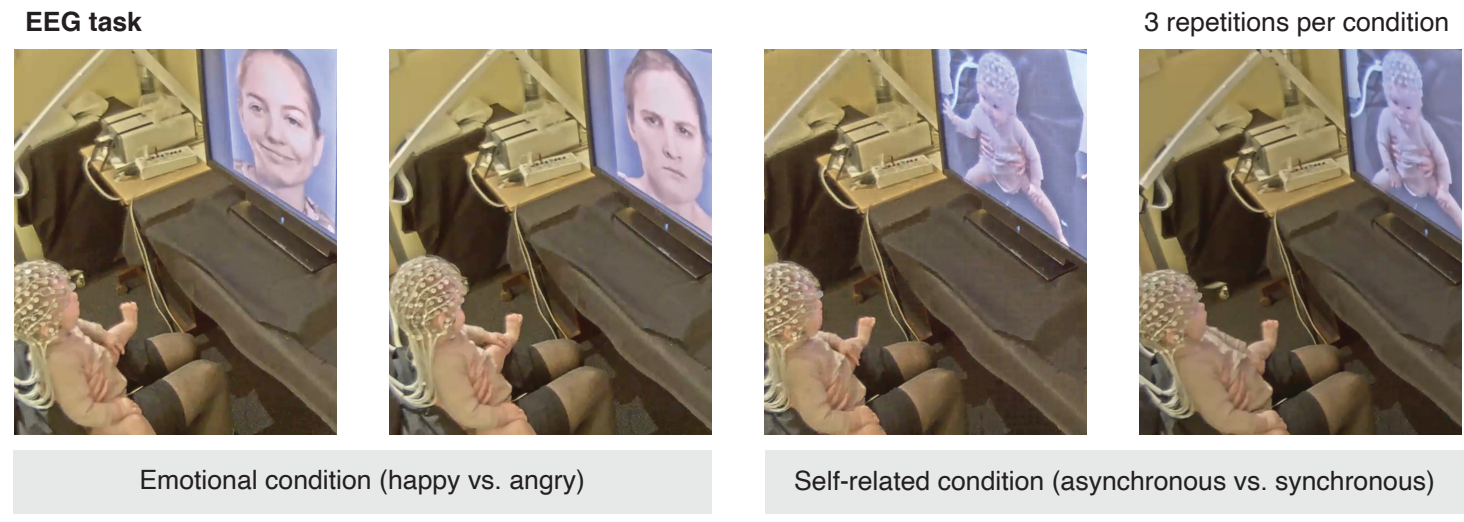

Note. Infants were seated on the parent's lap in front of a display. They saw moving happy and angry faces in the emotional conditions, and real-time or delayed videos of themselves in the self-related condition. Each stimulus lasted for $20 \mathrm{~s}$ and was repeated maximally three times.

Measures. The EEG was recorded from a 128-channel Geodesic Sensor Net with a NetAmps 300 amplifier (Electrical Geodesics Inc., Eugene, OR, USA, Figure 3) at a sampling rate of $500 \mathrm{~Hz}$. Data were recorded with reference to the vertex and online bandpass filtered between 0.01 and $100 \mathrm{~Hz}$. The impedance of all electrodes was kept below $50 \mathrm{k} \Omega$. Concurrent ECG was recorded with a 2-electrode setup, one on the lowest left rib, one on the right clavicle, with a Polygraph Input Box (Electrical Geodesics Inc., Eugene, OR, USA), connected to the same NetAmps 300 amplifier.

Looking time during stimulus presentation was assessed with two cameras, one filming the infant's face, and another one filming the stimuli on the screen. Looking duration was coded as the time that the infant's gaze was directed to the screen. 


\section{Infant Behavior Questionnaire}

At the end of the first session, caregivers completed the Infant Behavior

Questionnaire - Revised - Very Short Form (IBQ-R), a 37-item questionnaire that assessed three broad subscales of infant temperament (Putnam et al., 2014). Responses were given on a 7-point Likert scale, and subscale scores for Surgency, Negative Affect, and Effortful

Control were calculated as the mean over the items belonging to the subscale.

\section{Figure 3}

Channel Layout of the 128-Channel Geodesic Sensor Net

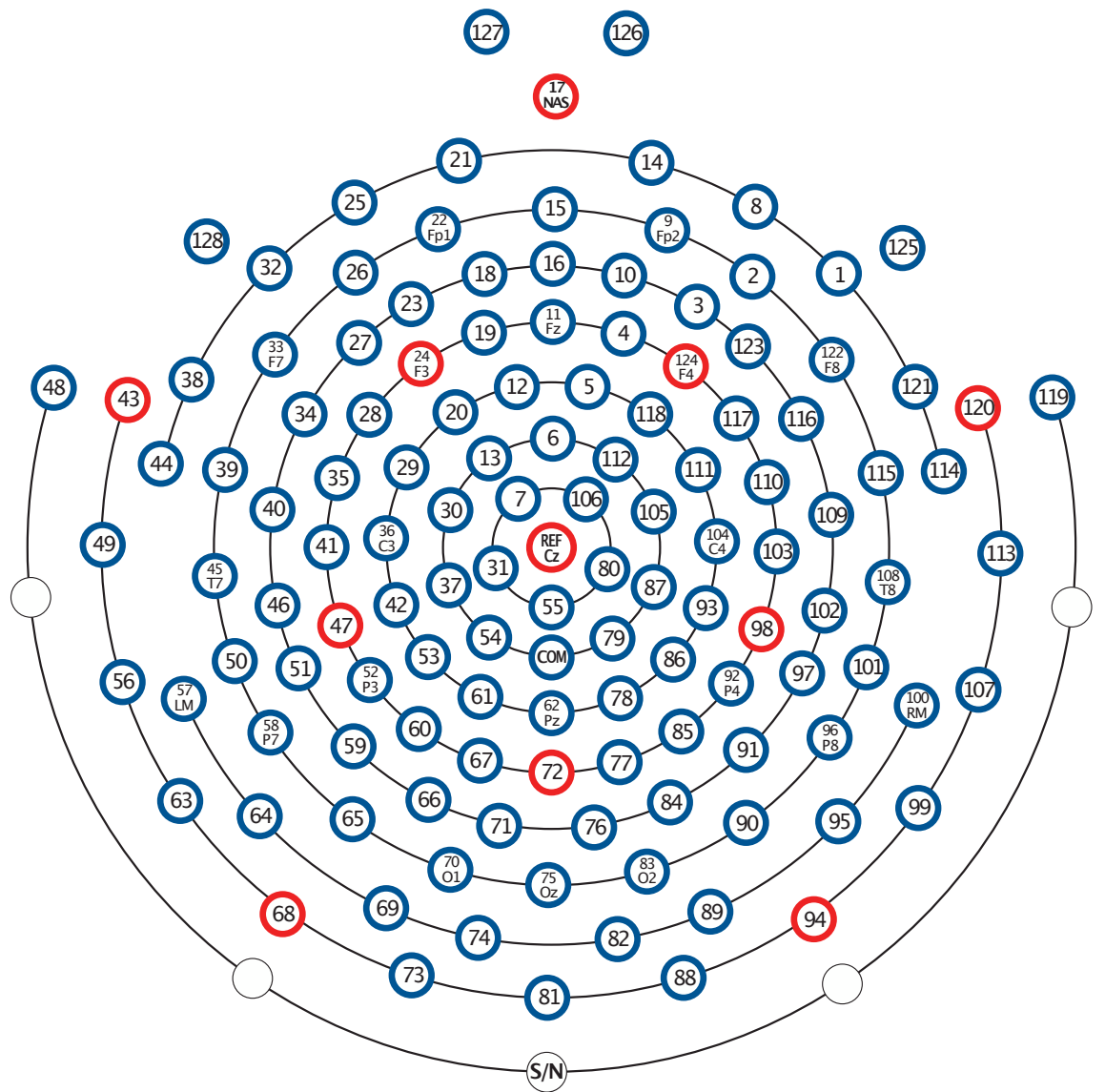

Note. The outer electrodes (E43, E48, E49, E56, E63, E68, E73, E81, E88, E94, E99, E107, E113, E119, E120, E125, E126, E127, E128) were removed from the analyses. 


\section{Data Processing}

\section{Looking-Time Preprocessing}

As a first step, the ECG and stimulus presentation during the looking-time task was assessed to include trials. For synchronous trials, the ECG had to match with $85 \%$ of the recorded R-peaks. In synchronous trials, this meant that sometimes a beat was skipped, because no R-peak was detected. In asynchronous trials, some R-peaks were not detected, and thus, the stimulus did not light up, if the speed was set to $110 \%$, the trials had to be between $85 \%$ and $130 \%$ of the actual heartrate, and if the speed was set to $90 \%$, the trials had to be between $70 \%$ and $115 \%$ of the actual heartrate. These percentages were chosen based on the errors that could occur in the recording of three interbeat intervals at the beginning of the asynchronous trial. Important to note is that a speed of $100 \%$ in the asynchronous trial meant that just the speed was similar as the recorded heartrate, but the presented beats still did not coincide with the recorded R-peaks, unlike in the synchronous trials.

In the second step, valid trials were selected based on the monitoring videos. Looking time and other behavior during stimulus presentation was manually coded. Trials were excluded if there were distractions from the parent, experimenter, or other reasons in the room or the infant cried for more than $5 \mathrm{~s}$. Finally, looking time had to be at least $5 \mathrm{~s}$ per trial (Maister et al., 2017). A block was included if both the synchronous and asynchronous stimulus presentation met all the criteria above. This resulted in an average inclusion of $2.4 \pm$ 1.9 blocks in the bimodal condition, and $5.9 \pm 3.8$ blocks in the trimodal condition.

\section{Heartbeat Evoked Potentials Preprocessing}

EEG and ECG recordings were preprocessed using Matlab 2017b and EEGLAB (version 14.1.1b; Delorme \& Makeig, 2004). The outer electrodes (E43, E48, E49, E56, E63, E68, E73, E81, E88, E94, E99, E107, E113, E119, E120, E125, E126, E127, E128, Figure 3) were removed from the data due to bad contact with the scalp. R-peaks in the ECG were 
detected with the EEGLAB extension HEPLAB (Perakakis, 2019) using the ECGLAB slow algorithm (de Carvalho, da Rocha, de Oliveira Nascimento, Neto, \& Junqueira, 2002). Automatic R-peak detection was followed by a visual inspection and manual correction to ensure appropriate R-peak detection. EEG data were filtered with a $0.3-30 \mathrm{~Hz}$ bandpass filter. Bad channels were detected using visual inspection, and replaced using spherical interpolation. The data were re-referenced to the average reference. Afterwards, data were segmented in $20 \mathrm{~s}$ long segments, that corresponded to the four conditions. Epochs from -100 to $350 \mathrm{~ms}$ relative to the R-peak were extracted for each condition, and epochs were rejected using a semiautomatic approach. Firstly, all epochs containing multiple R-peaks (RR interval $<350 \mathrm{~ms}$ ) were rejected, as to avoid contamination with the cardiac field artefact of the subsequent heartbeat. Then, epochs where one or more electrodes exceeded an amplitude of \pm $250 \mu \mathrm{V}$ were rejected. Finally, the datasets were visually inspected for further artefacts. For statistical analyses, a grand average over all epochs within each condition calculated (see Table 1 for number of epochs included in each condition).

\section{Table 1}

Epoch Count per Condition

\begin{tabular}{ll}
\hline Condition & Mean number of epochs (SD) \\
\hline Angry & $70.3(22.2)$ \\
Happy & $63.0(28.3)$ \\
Self Synchronous & $55.4(25.6)$ \\
Self Asynchronous & $69.2(32.9)$ \\
\hline
\end{tabular}




\section{Results}

\section{Behavioral task}

A Bayesian multilevel model was used to analyze the looking-time data. The model was implemented in Stan (Carpenter et al., 2017) using the R-package brms (Bürkner, 2017). Samples of posterior probability distributions for all estimated parameters were drawn with a Hamiltonian Monte Carlo sampling algorithm. Four independent Markov chains, each with 1000 warm-up samples, followed 3000 samples from the posterior distribution were used. The final 3000 samples of each chain were used for statistical inference. Minimally informative priors were used for all parameters of the model (normal distribution with $M=0$, and $S D=10$ ). Convergence was confirmed with $\mathrm{R}$ hat statistics that were $<1.1$ for all estimates. The $95 \%$ credible intervals (CI) of the posterior distributions were interpreted as the possible range of the parameter given the data, and existence of an effect was inferred when the CI did not contain 0. For the Bayesian multilevel model, a random intercept was modeled for each participant. Additionally, main effects synchrony (synchronous vs. asynchronous) and modality (bimodal vs. trimodal) were modeled. The model was specified as following (lookingtime $\sim$ synchrony + modality $+(1 \mid$ participant.ID)).

The Bayesian multilevel model showed no difference in looking time between synchronous and asynchronous stimuli $(b=0.54, \mathrm{CI}=[-0.15: 1.22])$. There was a significant effect of modality $(b=-2.04, \mathrm{CI}=-2.74 ;-1.33])$, showing that infants looked longer at trimodal $(M P E=12.0, C I=[11.6 ; 12.7])$ than bimodal stimuli $(M P E=10.0, C I=[9.3$; 10.7], Figure 4).

In the bimodal condition, 15 infants looked longer at synchronous, and 9 infants longer at asynchronous stimuli. A Bayesian binomial test showed that the estimated frequency of longer looking times at synchronous stimuli (0.62), was not significantly 
different from the 0.5 chance level $(\mathrm{CI}=[0.43,0.79])$. In the trimodal condition, 11 infants looked longer at synchronous, and 13 infants longer at synchronous stimuli, the binomial test again showed that this was not significantly different from chance (estimated frequency of longer looking times at synchronous stimuli: $0.46 ; \mathrm{CI}=[0.28-0.65])$.

We furthermore assessed whether interoceptive sensitivity, defined as the difference in looking time between synchronous and asynchronous stimulated was correlated with the subscales of the IBQ-R (responses to each of the IBQ-R subscales are summarized in Table 2). To this end, we performed Bayesian correlation analyses using the BayesFactor package in R. None of the three subscales of the IBQ-R showed a significant correlation with interoceptive sensitivity (Table 3).

Table 2

Responses to the IBQ-R

\begin{tabular}{lc}
\hline IBQ-R Subscale & Mean (SD) \\
\hline Surgency & $4.91(0.68)$ \\
Negative affect & $3.60(0.96)$ \\
Effortful control & $4.82(0.59)$ \\
\hline
\end{tabular}

Table 3

Results of the Bayesian Correlation Analyses of Interoceptive Sensitivity with the IBQ-R

\begin{tabular}{llll}
\hline IBQ-R Subscale & rho & $95 \%$ CI & $\mathrm{BF}_{10}$ \\
\hline Surgency & -0.19 & $-0.49 ; 0.14$ & 0.75 \\
Negative Affect & -0.08 & $-0.41 ; 0.24$ & 0.46 \\
Effortful Control & -0.22 & $-0.52 ; 0.11$ & 0.93 \\
\hline
\end{tabular}




\section{Figure 4}

Raincloud Plots of Looking Time in Each Condition

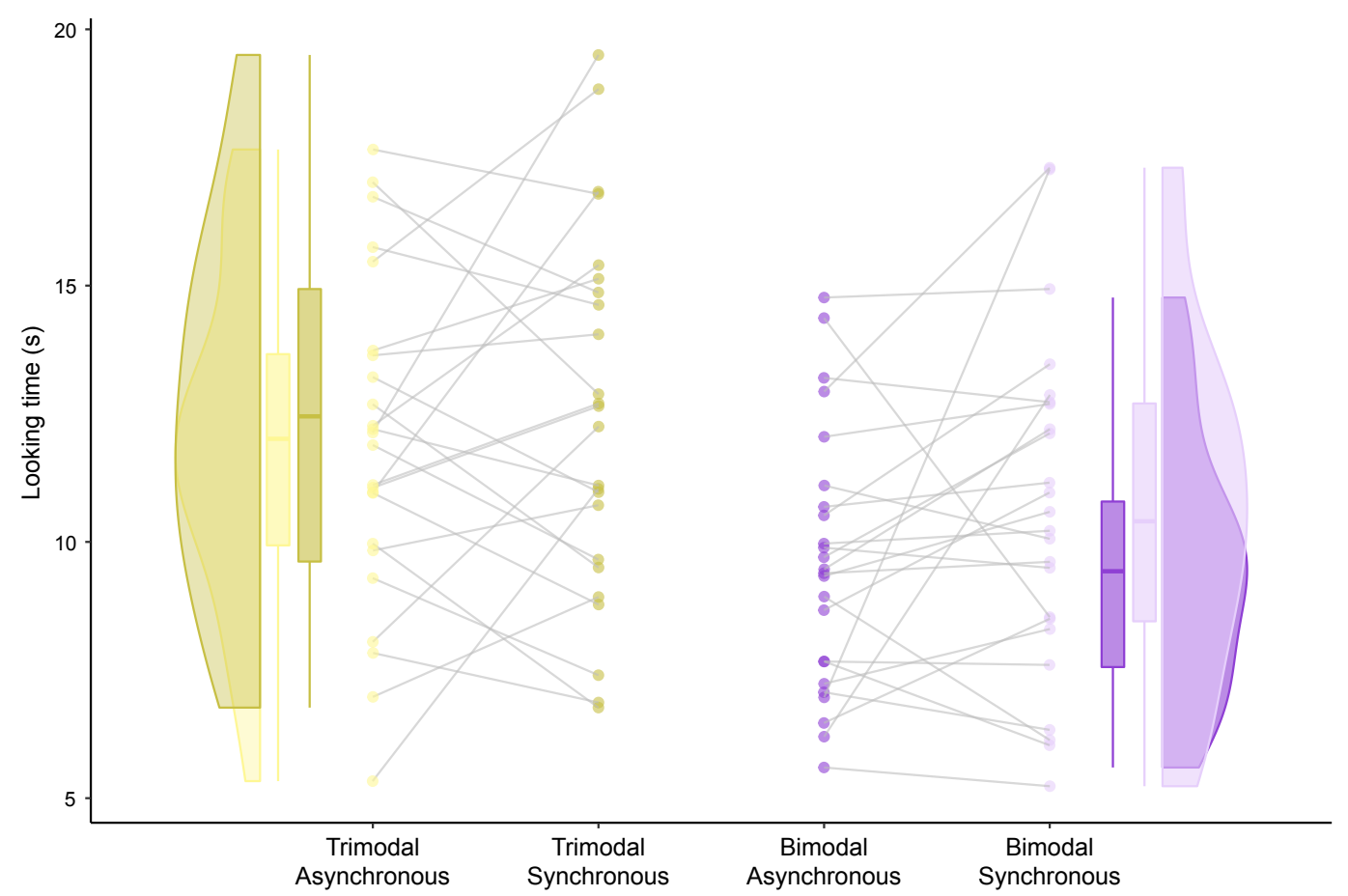

Note. As marked by the asterisk, there was a significantly longer looking time in the trimodal than the bimodal conditions. Colors indicate the different conditions and dots the individual observations. Boxes indicate the interquartile ranges, horizontal lines mark the medians, and whiskers indicate the lower and upper extremes.

\section{HEP}

As the HEP is a cortical potential that has been reported to be highly heterogeneous in terms of topography and latency (Coll et al., 2021; Park \& Blanke, 2019), we performed cluster-based permutation t-tests implemented in FieldTrip (Maris \& Oostenveld, 2007; Oostenveld, Fries, Maris, \& Schoffelen, 2011) to assess for any differences between experimental conditions, while controlling for multiple comparisons across space and time. This procedure clusters adjacent spatiotemporal data points for which t-values exceeded a cluster threshold p-value of .05 (two-sided). Cluster statistics are calculated as the sum of the 
t-values of all points within a spatiotemporal cluster. This cluster statistic was then evaluated under the cluster-distribution under the null hypothesis. Condition labels were randomly shuffled 1000 times, and maximum cluster-level statistics were retained for each permutation. The two-tailed Monte-Carlo p-value reflects the proportion of elements in the distribution that exceeded the observed maximum cluster-level statistic, and was considered significant $<.05$. Neighbors distance of $4 \mathrm{~cm}$, on average 6.3 neighbors per channel. Cluster based permutation t-tests did not show any significant differences in the HEP between conditions for both the emotional context and the self-related context.

Additional control analyses, to assess whether looking duration differed between the conditions in the EEG task, using Bayesian multilevel models with the same parameters as described above, did not show a significant difference in looking time between angry and happy faces $(b=-0.39, C I=[-1.79: 1.01])$. Neither was there a significant difference in looking time between the self-synchronous, and self-asynchronous conditions $(b=-0.12, \mathrm{CI}$ $=[-1.26: 1.05]$; looking durations are reported in Table 4).

Finally, to assess whether HEP amplitude, independent of experimental condition correlated with any of the subscales of the IBQ-R, or interoceptive sensitivity defined as the looking-time difference between synchronous and asynchronous stimuli in the looking-time task, cluster-based permutation correlation analyses were performed on the HEP averaged per participant across all conditions with each of the subscales of the IBQ-R. The HEP across all conditions did not correlate with any of the subscales of the IBQ-R. Also, no significant clusters for a correlation of the HEP and looking-time difference were found. 


\section{Table 4}

Looking Duration in Each of the EEG Conditions

\begin{tabular}{ll}
\hline Condition & Mean (SD) in s \\
\hline Self-synchronous & $12.2(5.0)$ \\
Self-asynchronous & $12.3(4.5)$ \\
Happy & $11.6(5.3)$ \\
Angry & $12.0(5.4)$ \\
\hline
\end{tabular}

\section{Discussion}

In this study, we investigated interoception and its link to emotion and self-awareness in infants aged 5-7 months. Contrary to our prediction, we did not find conclusive evidence for a systematic looking preference towards either asynchronous or synchronous stimuli while we confirmed a clear preference for trimodal as compared to bimodal stimuli. Additionally, and again unlike predicted, we did not observe any differences in the HEP across either varying emotional or self-related contexts, nor did the HEP correlate with parent's reports of infant temperament or the individual differences in looking time. Taken together, these results do neither support our hypotheses, nor are they in line with previous evidence of interoceptive sensitivity in infants recorded in a similar experimental setup (Maister et al., 2017). We can currently only speculate about the reason for these null findings.

In the looking-preference task, we found that infants looked longer at trimodal (audiovisuocardiac) than bimodal (visuocardiac) stimuli. This preference was not modulated by synchrony and confirms the validity of our looking-time preference measures. The availability of more sensory information lead to an increase in attentional resources dedicated to these stimuli (Bahrick \& Lickliter, 2000). We did however not replicate the finding that 
infants distinguish in looking time between stimuli that were either in- or out of synchrony with their own heartbeat (Maister et al., 2017). This lack of consistency could speculatively be linked both to subtle differences in the experimental paradigm, or to age differences.

In our study, we manually coded looking time, Maister and colleagues (2017) used eye tracking and gaze-controlled stimulus presentation, which may have increased sensitivity. We also used blinking objects, that were accompanied by a beep in the trimodal condition, contrastingly, Maister and colleagues (2017) used stimuli that moved up and down and were accompanied by an acoustic signal. It may have been the case that the difference between synchronous and asynchronous blinking stimuli was not salient enough to observe a lookingtime difference, which however questions the generalizability of previous data. Alternatively, the use of both a bimodal and trimodal condition may have interfered with synchrony detection, even though the presentation was counterbalanced, thus masking a potential effect due to our experimental design.

Another explanation could be found in the age difference between the two studies. Our sample was slightly older, which may have affected contingency detection. In paradigms using other sensory modalities discrimination ability emerges at different ages, and might even switch between a preference for contingent first, and then for non-contingent (Jacquey et al., 2020). Recent theories of interoceptive development state its importance for effective infant-caregiver interactions to maintain homeostasis (Ciaunica \& Crucianelli, 2019; Filippetti, 2021; Fotopoulou \& Tsakiris, 2017). This could link to the idea that while contingency detection of movement-related senses or of external stimuli (i.e. proprioception or touch) increases with increasing age (Rochat, 2003), the link between external and interoceptive signals might decrease, which could be demonstrated by decreasing attention to bodily signals. This hypothesis would be in line with the idea that when the infant grows 
older and motor skills develop, feeding interactions become more efficient (Filippetti, 2021), and instead attention is directed to the exploration of the world (Adolph \& Hoch, 2019).

Additionally, we did not find neurophysiological evidence for a modulation of interoceptive processing in infants across emotional or self-related contexts. A potential reason for that could be that we tested for differences in HEP amplitude while altering the emotional or self-related context by visual means only, it could be the case that the stimuli used were not salient enough to observe changes in the HEP. Emotion perception appears to be driven by perception of voice rather than facial expression in early infancy (Caron, Caron, \& MacLean, 1988; Flom \& Bahrick, 2007). It has been demonstrated that 4-month-old infants only discriminated affective facial expression when voice and face were presented in synchrony, but not when multimodal stimuli were presented asynchronously, or unimodal auditory or visual stimuli were presented (Flom \& Bahrick, 2007). Sensitivity to visual affective stimuli only emerged at 7-months. The infants tested in the current study were between 5 and 7 months old, and thus, the ability to discriminate between different emotional facial expressions from unimodal visual stimuli might not be fully developed, which could explain why we did not observe a difference in HEP. Still, this would not explain the difference to previous findings in a very similar setup using visual stimuli only (Maister et al., 2017). Nevertheless it would be interesting, to use auditory, or audiovisual emotional stimuli (Caron et al., 1988; Flom \& Bahrick, 2007).

Such a similar reasoning might be the case of the self-related stimuli. Even though, 5month-old infants have been reported to distinguish between synchronous and asynchronous displays of their leg movements (Bahrick \& Watson, 1985), these results are not consistent and rapidly developing (Zmyj, Hauf, \& Striano, 2009). Furthermore, while contingency perception has been discussed as an important precursor for self-recognition at a later age, these two concepts do not directly correlate (Klein-Radukic \& Zmyj, 2020). Furthermore, for 
explicit self-recognition, which has been found to modulate interoception in adults (Ainley et al., 2013, 2012), recognition of one's own facial features seems an additional driving force in addition to multisensory contingency, which only emerges later in infancy (Filippetti \& Tsakiris, 2018), and therefore contingency detection alone might not directly modulate the HEP. Longitudinal studies, relating cardiac interoception in infants to development of affect and self-awareness, would be required to experimentally address whether and how these concepts relate and potentially predict behavioral outcomes. To further study how interoception relates to behavioral outcomes, future studies should address the long-term stability and reliability of interoceptive measures. In adults, poor reliability of the HEP across experimental sessions has been reported (Verdonk et al., 2021), and behavioral measures show similar variability across timepoints in children (Ferentzi, Drew, Tihanyi, \& Köteles, 2018). While such variability could partially be explained by developmental changes across childhood (Ferentzi et al., 2018), the long-term stability and reliability of these measures requires urgent investigation to better understand the developmental trajectory of interoception.

Another potential limitation might be the case that our experimental design did not provide sufficient power and a high enough signal to noise ratio to detect the effects of interest in the HEP, despite at least as many repetitions as in previous studies (Maister et al., 2017). The HEP is a highly variable neural potential, that has been found across different latencies and topographies in adults (Coll et al., 2021). Furthermore, large amount of trials, or R-peaks, up to ten times higher as in the current experiment, are recommended to obtain reliable results in adult studies (Park \& Blanke, 2019). To increase the number of trials as much as possible, we opted for presenting the stimulus on screen for 20 seconds, without having a gaze-controlled stimulus presentation. While looking time was comparable between all conditions, we cannot ensure that the infant looked at the screen for the full 20 second 
period, and thus, there may have been periods in which no awareness of the self-related or emotional stimuli was present. Together with the short duration of the EEG recording, due to the limitations of experiments with infants, this might not have provided enough trials to find a reliable HEP.

\section{Outlook}

The growing literature on interoception development emphasizes its importance in relation to development of psychopathology (Murphy, Brewer, Catmur, \& Bird, 2017), and highlights its role in development of self-awareness and social cognition (Ciaunica \& Crucianelli, 2019; Filippetti, 2021; Fotopoulou \& Tsakiris, 2017). There is however a gap between theoretical discussions of interoceptive development and empirical evidence. More studies, using larger samples would be required to assess generalizability of the null results reported in our study, and positive findings reported earlier (Maister et al., 2017). While assessments of interoceptive awareness at a single timepoint are a start to better understand how interoception develops, longitudinal studies and investigations addressing the reliability and long-term stability of the measures used would be required to obtain a broader understanding of the implications of interoception in early life. Aligning theory and empirical data, by investigating how interoception relates to the development of emotion regulation and self-awareness across infancy and childhood longitudinally, might provide a more complete understanding of the implications interoception in psychopathology from a developmental perspective. 


\section{References}

Adolph, K. E., \& Hoch, J. E. (2019). Motor development: Embodied, embedded, enculturated, and enabling. Annual Review of Psychology, 70(1), 141-164. https://doi.org/10.1146/annurev-psych-010418-102836

Ainley, V., Maister, L., Brokfeld, J., Farmer, H., \& Tsakiris, M. (2013). More of myself: Manipulating interoceptive awareness by heightened attention to bodily and narrative aspects of the self. Consciousness and Cognition, 22(4), 1231-1238. https://doi.org/10.1016/j.concog.2013.08.004

Ainley, V., Tajadura-Jiménez, A., Fotopoulou, A., \& Tsakiris, M. (2012). Looking into myself: Changes in interoceptive sensitivity during mirror self-observation. Psychophysiology, 49. https://doi.org/10.1111/j.1469-8986.2012.01468.x

Amsterdam, B. (1972). Mirror self-image reactions before age two. Developmental Psychobiology, 5(4), 297-305.

Aspell, J. E., Heydrich, L., Marillier, G., Lavanchy, T., Herbelin, B., \& Blanke, O. (2013). Turning body and self inside out: Visualized heartbeats alter bodily selfconsciousness and tactile perception. Psychological Science, 24(12), 2445-2453.

Azzalini, D., Rebollo, I., \& Tallon-Baudry, C. (2019). Visceral signals shape brain dynamics and cognition. Trends in Cognitive Sciences, 23(6), 488-509. https://doi.org/10.1016/j.tics.2019.03.007

Babo-Rebelo, M., Buot, A., \& Tallon-Baudry, C. (2019). Neural responses to heartbeats distinguish self from other during imagination. NeuroImage. https://doi.org/10.1016/j.neuroimage.2019.02.012

Bahrick, L. E. (2013). Body perception: Intersensory origins of self and other perception in newborns. Current Biology, 23(23), R1039-R1041. https://doi.org/10.1016/j.tcb.2013.07.003 
Bahrick, L. E., \& Lickliter, R. (2000). Intersensory redundancy guides attentional selectivity and perceptual learning in infancy. Developmental Psychology, 36(2), 190-201. (2000-13792-005). https://doi.org/10.1037/0012-1649.36.2.190

Bahrick, L. E., \& Lickliter, R. (2012). The role of intersensory redundancy in early perceptual, cognitive, and social development. In A. J. Bremner, D. J. Lewkowicz, \& C. Spence (Eds.), Multisensory development (pp. 183-205). Oxford, England: Oxford University Press.

Bahrick, L. E., \& Watson, J. S. (1985). Detection of intermodal proprioceptive-visual contingency as a potential basis of self-perception in infancy. Developmental Psychology, 21(6), 963-973. (1986-08693-001). https://doi.org/10.1037/00121649.21.6.963

Bürkner, P.-C. (2017). brms: An r package for bayesian multilevel models using stan. Journal of Statistical Software, 80(1). https://doi.org/10.18637/jss.v080.i01

Caron, A. J., Caron, R. F., \& MacLean, D. J. (1988). Infant Discrimination of Naturalistic Emotional Expressions: The Role of Face and Voice. Child Development, 59(3), 604616.

Carpenter, B., Gelman, A., Hoffman, M. D., Lee, D., Goodrich, B., Betancourt, M., ... Riddell, A. (2017). Stan: A Probabilistic Programming Language. Journal of Statistical Software, 76(1). https://doi.org/10.18637/jss.v076.i01

Ciaunica, A., \& Crucianelli, L. (2019). Minimal self-awareness from within: A developmental perspective. Journal of Consciousness Studies, 26(3-4), 207-226.

Coll, M.-P., Hobson, H., Bird, G., \& Murphy, J. (2021). Systematic review and meta-analysis of the relationship between the heartbeat-evoked potential and interoception. Neuroscience \& Biobehavioral Reviews, 122, 190-200. https://doi.org/10.1016/j.neubiorev.2020.12.012 
Craig, A. D. (2002). How do you feel? Interoception: the sense of the physiological condition of the body. Nature Reviews Neuroscience, 3(8), 655-667.

Critchley, H. D., \& Garfinkel, S. N. (2017). Interoception and emotion. Current Opinion in Psychology, 17, 7-14. https://doi.org/10.1016/j.copsyc.2017.04.020

de Carvalho, J. L. A., da Rocha, A. F., de Oliveira Nascimento, F. A., Neto, J. S., \& Junqueira, L. F. (2002). Development of a Matlab software for analysis of heart rate variability. 6th International Conference on Signal Processing, 2002., 2, 1488-1491. Beijing, China: IEEE. https://doi.org/10.1109/ICOSP.2002.1180076

Delorme, A., \& Makeig, S. (2004). EEGLAB: An open source toolbox for analysis of singletrial EEG dynamics including independent component analysis. Journal of Neuroscience Methods, 134(1), 9-21. https://doi.org/10.1016/j.jneumeth.2003.10.009

Ferentzi, E., Drew, R., Tihanyi, B. T., \& Köteles, F. (2018). Interoceptive accuracy and body awareness - Temporal and longitudinal associations in a non-clinical sample. Physiology \& Behavior, 184, 100-107. https://doi.org/10.1016/j.physbeh.2017.11.015

Filippetti, M. L. (2021). Being in tune with your body: The emergence of interoceptive processing through caregiver-infant feeding interactions. Child Development Perspectives, 15(3), 182-188. https://doi.org/10.1111/cdep.12420

Filippetti, M. L., Johnson, M. H., Lloyd-Fox, S., Dragovic, D., \& Farroni, T. (2013). Body perception in newborns. Current Biology, 23(23), 2413-2416. https://doi.org/10.1016/j.cub.2013.10.017

Filippetti, M. L., \& Tsakiris, M. (2018). Just before i recognize myself: The role of featural and multisensory cues leading up to explicit mirror self-recognition. Infancy. https://doi.org/10.1111/infa.12236

Flasbeck, V., Popkirov, S., Ebert, A., \& Brüne, M. (2020). Altered interoception in patients with borderline personality disorder: A study using heartbeat-evoked potentials. 
Borderline Personality Disorder and Emotion Dysregulation, 7(1), 24.

https://doi.org/10.1186/s40479-020-00139-1

Flom, R., \& Bahrick, L. E. (2007). The development of infant discrimination of affect in multimodal and unimodal stimulation: The role of intersensory redundancy. Developmental Psychology, 43(1), 238-252. https://doi.org/10.1037/00121649.43.1.238

Fotopoulou, A., \& Tsakiris, M. (2017). Mentalizing homeostasis: The social origins of interoceptive inference. Neuropsychoanalysis, 19(1), 3-28. https://doi.org/10.1080/15294145.2017.1294031

Gentsch, A., Sel, A., Marshall, A. C., \& Schütz-Bosbach, S. (2019). Affective interoceptive inference: Evidence from heart-beat evoked brain potentials. Human Brain Mapping, 40(1), 20-33. https://doi.org/10.1002/hbm.24352

Heydrich, L., Aspell, J. E., Marillier, G., Lavanchy, T., Herbelin, B., \& Blanke, O. (2018). Cardio-visual full body illusion alters bodily self-consciousness and tactile processing in somatosensory cortex. Scientific Reports, 8(1), 9230. https://doi.org/10.1038/s41598-018-27698-2

Jacquey, L., Fagard, J., Esseily, R., \& O’Regan, J. K. (2020). Detection of sensorimotor contingencies in infants before the age of 1 year: A comprehensive review. Developmental Psychology, 56(7), 1233-1251. https://doi.org/10.1037/dev0000916

Klein-Radukic, S., \& Zmyj, N. (2020). No evidence for infants' contingency detection as a precursor of toddlers' self-recognition. Infant Behavior and Development, 60, 101459. https://doi.org/10.1016/j.infbeh.2020.101459

Luft, C. D. B., \& Bhattacharya, J. (2015). Aroused with heart: Modulation of heartbeat evoked potential by arousal induction and its oscillatory correlates. Scientific Reports, 5(1). https://doi.org/10.1038/srep15717 
Maister, L., Tang, T., \& Tsakiris, M. (2017). Neurobehavioral evidence of interoceptive sensitivity in early infancy. ELife, 6, e25318. https://doi.org/10.7554/eLife.25318

Maris, E., \& Oostenveld, R. (2007). Nonparametric statistical testing of EEG- and MEG-data. Journal of Neuroscience Methods, 164(1), 177-190.

https://doi.org/10.1016/j.jneumeth.2007.03.024

Miyazaki, M., \& Hiraki, K. (2006). Delayed intermodal contingency affects young children's recognition of their current self. Child Development, 77(3), 736-750.

https://doi.org/10.1111/j.1467-8624.2006.00900.x

Montirosso, R., \& McGlone, F. (2020). The body comes first. Embodied reparation and the co-creation of infant bodily-self. Neuroscience \& Biobehavioral Reviews, 113, 77-87. https://doi.org/10.1016/j.neubiorev.2020.03.003

Mundy, P., \& Jarrold, W. (2010). Infant joint attention, neural networks and social cognition. Neural Networks, 23(8-9), 985-997. https://doi.org/10.1016/j.neunet.2010.08.009

Murphy, J., Brewer, R., Catmur, C., \& Bird, G. (2017). Interoception and psychopathology: A developmental neuroscience perspective. Developmental Cognitive Neuroscience, 23, 45-56. https://doi.org/10.1016/j.den.2016.12.006

Oostenveld, R., Fries, P., Maris, E., \& Schoffelen, J.-M. (2011). Fieldtrip: Open source software for advanced analysis of MEG, EEG, and invasive electrophysiological data. Computational Intelligence and Neuroscience, 2011, 1-9. https://doi.org/10.1155/2011/156869

Park, H.-D., \& Blanke, O. (2019). Heartbeat-evoked cortical responses: Underlying mechanisms, functional roles, and methodological considerations. NeuroImage, 197, 502-511. https://doi.org/10.1016/j.neuroimage.2019.04.081 
Perakakis, P. (2019). HEPLAB: a Matlab graphical interface for the preprocessing of the heartbeat-evoked potential (Version Version v1.0.0). Zenodo. Retrieved from http://doi.org/10.5281/zenodo.2649943

Petzschner, F. H., Weber, L. A., Wellstein, K. V., Paolini, G., Tri Do, C., \& Stephan, K. E. (2019). Focus of attention modulates the heartbeat evoked potential. NeuroImage, 186. https://doi.org/10.1101/384305

Petzschner, Frederike H., Garfinkel, S. N., Paulus, M. P., Koch, C., \& Khalsa, S. S. (2021). Computational models of interoception and body regulation. Trends in Neurosciences, 44(1), 63-76. https://doi.org/10.1016/j.tins.2020.09.012

Putnam, S. P., Helbig, A. L., Gartstein, M. A., Rothbart, M. K., \& Leerkes, E. (2014). Development and assessment of short and very short forms of the infant behavior questionnaire-revised. Journal of Personality Assessment, 96(4), 445-458. https://doi.org/10.1080/00223891.2013.841171

Rochat, P. (2003). Five levels of self-awareness as they unfold early in life. Consciousness and Cognition, 12(4), 717-731. https://doi.org/10.1016/S1053-8100(03)00081-3

Rochat, P., \& Botto, S. V. (2021). From implicit to explicit body awareness in the first two years of life. In P. Rochat \& S. V. Botto, Body Schema and Body Image (pp. 181193). Oxford University Press. https://doi.org/10.1093/oso/9780198851721.003.0011

Rochat, P., \& Striano, T. (2002). Who's in the mirror? Self-other discrimination in specular images by four-and nine-month-old infants. Child Development, 73(1), 35-46.

Schulz, A., Köster, S., Beutel, M. E., Schächinger, H., Vögele, C., Rost, S., ... Michal, M. (2015). Altered patterns of heartbeat-evoked potentials in depersonalization/derealization disorder: Neurophysiological evidence for impaired cortical representation of bodily signals. Psychosomatic Medicine, 77(5), 506-516. https://doi.org/10.1097/PSY.0000000000000195 
Simon, D., Craig, K. D., Gosselin, F., Belin, P., \& Rainville, P. (2008). Recognition and discrimination of prototypical dynamic expressions of pain and emotions. Pain, 135(1), 55-64. https://doi.org/10.1016/j.pain.2007.05.008

Tsakiris, M. (2017). The multisensory basis of the self: From body to identity to others. Quarterly Journal of Experimental Psychology, 70(4), 597-609. https://doi.org/10.1080/17470218.2016.1181768

Verdonk, C., Trousselard, M., Di Bernardi Luft, C., Medani, T., Billaud, J., Ramdani, C., ... Vialatte, F. (2021). The heartbeat evoked potential does not support strong interoceptive sensibility in trait mindfulness. Psychophysiology, 58(10). https://doi.org/10.1111/psyp.13891

Zmyj, N., Hauf, P., \& Striano, T. (2009). Discrimination between real-time and delayed visual feedback of self-performed leg movements in the first year of life. Cognition, Brain, and Behavior, 12.

Zmyj, N., Jank, J., Schütz-Bosbach, S., \& Daum, M. M. (2011). Detection of visual-tactile contingency in the first year after birth. Cognition, 120(1), 82-89. https://doi.org/10.1016/j.cognition.2011.03.001 